\title{
Gender-Specific Association of ATP2B1 Variants with Susceptibility to Essential Hypertension in the Han Chinese Population
}

\author{
Jin Xu, ${ }^{1}$ Hai-xia Qian, ${ }^{1}$ Su-pei Hu, ${ }^{2}$ Li-ya Liu, ${ }^{1}$ Mi Zhou, ${ }^{1}$ Mei Feng, ${ }^{1}$ Jia Su, ${ }^{3}$ and Lin-dan Ji \\ ${ }^{1}$ Department of Preventive Medicine, School of Medicine, Ningbo University, Ningbo 315211, China \\ ${ }^{2}$ Department of Research and Teaching, Ningbo No. 2 Hospital, Ningbo 315010, China \\ ${ }^{3}$ Department of Gerontology, Ningbo No. 1 Hospital, Ningbo 315010, China \\ ${ }^{4}$ Department of Biochemistry, School of Medicine, Ningbo University, Ningbo 315211, China
}

Correspondence should be addressed to Lin-dan Ji; jilindan@nbu.edu.cn

Received 4 November 2015; Revised 10 December 2015; Accepted 20 December 2015

Academic Editor: Laurent Metzinger

Copyright (C) 2016 Jin Xu et al. This is an open access article distributed under the Creative Commons Attribution License, which permits unrestricted use, distribution, and reproduction in any medium, provided the original work is properly cited.

\begin{abstract}
Previous genome-wide association studies (GWASs) found that several ATP2B1 variants are associated with essential hypertension (EHT). But the "genome-wide significant" ATP2B1 SNPs (rs2681472, rs2681492, rs17249754, and rs1105378) are in strong linkage disequilibrium (LD) and are located in the same LD block in Chinese populations. We asked whether there are other SNPs within the ATP2B1 gene associated with susceptibility to EHT in the Han Chinese population. Therefore, we performed a case-control study to investigate the association of seven tagSNPs within the ATP2B1 gene and EHT in the Han Chinese population, and we then analyzed the interaction among different SNPs and nongenetic risk factors for EHT. A total of 902 essential hypertensive cases and 902 normotensive controls were involved in the study. All 7 tagSNPs within the ATP2B1 gene were retrieved from HapMap, and genotyping was performed using the Tm-shift genotyping method. Chi-squared test, logistic regression, and propensity score analysis showed that rs17249754 was associated with EHT, particularly in females. The MDR analysis demonstrated that the interaction of rs2070759, rs17249754, TC, TG, and BMI increased the susceptibility to hypertension. Crossover analysis and stratified analysis indicated that BMI has a major effect on the development of hypertension, while ATP2B1 variants have a minor effect.
\end{abstract}

\section{Introduction}

Because of its high prevalence and substantial impact on several cardiovascular diseases, hypertension is considered a major contributor to the global health burden [1]. Approximately $95 \%$ of hypertensive patients are diagnosed with essential hypertension (EHT), which is defined as high blood pressure (BP) with no identifiable cause [2]. EHT is one of the most common complex genetic disorders, with heritability ranging from $31 \%$ to $68 \%$ [3]. However, attempts to identify the genetic basis of EHT have been frequently unsuccessful and of relatively low yield [4]. The inability to identify the genetic basis of EHT may be due to the cumulative impact of multiple genes interacting with a variety of environmental factors in the pathogenesis of hypertension $[5,6]$.
In 2009, based on a genome-wide association study (GWAS) conducted by the Cohorts for Heart and Aging Research in Genome Epidemiology (CHARGE) Consortium, genetic polymorphisms of $A T P 2 B 1$ were found to be significantly related to systolic blood pressure (SBP), diastolic blood pressure (DBP), and hypertension [7]. These SNPs were also replicated in the European populations by the Global Blood Pressure Genetics (Global BPgen) Consortium [8]. Moreover, combined analysis of these two datasets further confirmed that only ATP2B1 variants reached genome-wide significance threshold $\left(P<5 \times 10^{-8}\right)$ with SBP (rs2681492), DBP (rs2681472), and hypertension (rs2681472) [9]. Similarly, in a study of the Korean Association Resource (KARE), rs17249754, which is located near the ATP2B1 gene, was found to be strongly associated with SBP [10]. Moreover, in a 


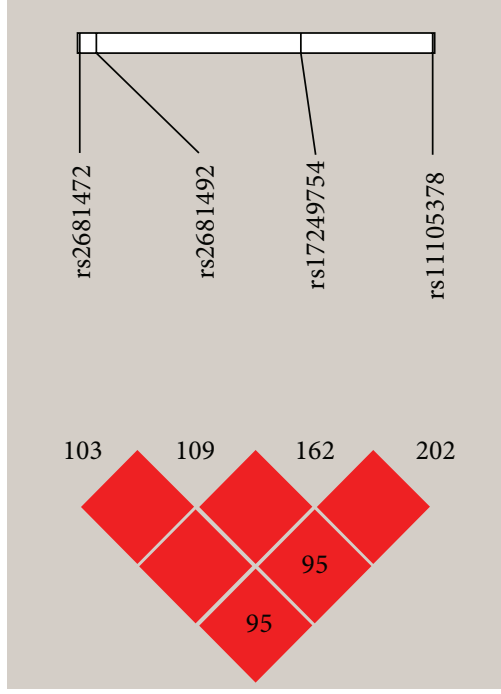

(a)

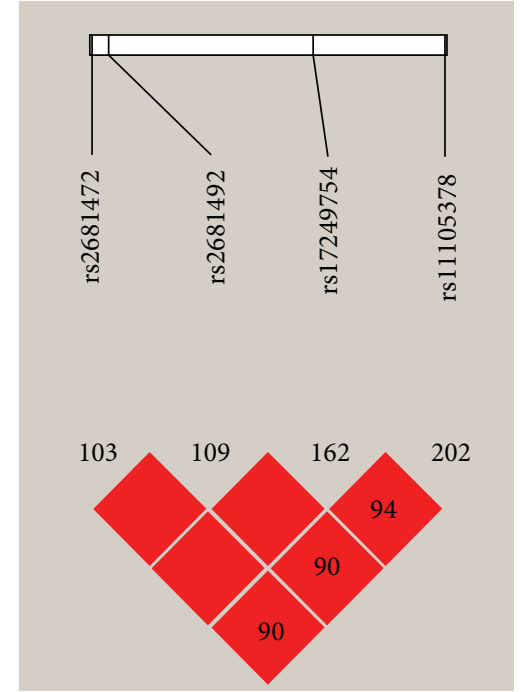

(b)

FIGURE 1: The patterns of linkage disequilibrium for 4 SNPs with $D^{\prime}$ (a) and $r^{2}$ (b).

study by the Japanese Millennium Genome Project, another $A T P 2 B 1$ variant, rs11105378, was found to have the most significant association with hypertension $\left(P=4.1 \times 10^{-11}\right)$, and the association was cross-validated by replication analysis with the Global BPgen dataset $\left(P=5.9 \times 10^{-4}\right)[1]$. Metaanalysis of GWASs in East Asians indicated that rs17249754 was associated with $\operatorname{SBP}\left(P=7.7 \times 10^{-20}\right)$ and $\operatorname{DBP}(P=$ $\left.1.9 \times 10^{-13}\right)[11]$.

Although ATP2B1 was confirmed to be associated with blood pressure or hypertension in various populations, the "significant" SNPs (rs2681472, rs2681492, rs17249754, and rs1105378) found in the GWASs are in strong linkage disequilibrium (LD) and are located in the same LD block (HapMap $\left.\mathrm{CHB} D^{\prime}>0.95, r^{2}>0.9\right)$ in the Chinese population (Figure 1). We wondered whether there are other SNPs within the ATP2B1 gene associated with the susceptibility to EHT in the Han Chinese population. In the current study, we conducted a replication analysis to test the association of seven tagSNPs within the ATP2B1 gene and EHT in the Han Chinese population. Subsequently, we analyzed the interaction among different SNPs and nongenetic risk factors for EHT, which provided additional information on the role of $A T P 2 B 1$ variants.

\section{Materials and Methods}

2.1. Ethics Statement. The protocol of this study was approved by the medical ethics committee of Ningbo University. The health records and blood samples of the participants were collected with informed written consent.

2.2. Study Participants. The details of the study participants have been described previously [12]. Briefly, we collected more than 10,000 health records from our established database of Ningbo Chronic Diseases Cohort. The participants in this database are 30 to 75 years old, Han Chinese, living in Ningbo City (East coast of China) for at least three generations without migration history. Patients with secondary hypertension, severe cardiovascular diseases, diabetes, kidney diseases, or other major chronic illnesses according to their health records were excluded before casecontrol paring. Hypertension in this study was defined as sitting systolic blood pressure (SBP) $\geq 140 \mathrm{mmHg}$ and/or diastolic blood pressure (DBP) $\geq 90 \mathrm{mmHg}$ or self-reported use of antihypertensive medication. Participants with SBP $\leq 120 \mathrm{mmHg}$ and DBP $\leq 80 \mathrm{mmHg}$ were recruited as controls. Subsequently, 902 hypertensive cases and 902 normotensive controls, matched for age and sex, were selected with informed consent.

2.3. Measurement of Clinical Parameters. With informed written consent, two milliliters of venous blood was collected with ethylene diamine tetraacetic acid as an anticoagulant. Subsequently, the serum levels of total cholesterol (TC), highdensity lipoprotein (HDL), and triglyceride (TG) were measured enzymatically using a Hitachi automatic biochemistry analyzer 7100. Clinical information, including body mass index (BMI), and weekly alcohol and cigarette consumption were also obtained. In this study, people who consumed $\geq 70 \mathrm{~g}$ of alcohol per week for more than 1 year were defined as individuals with alcohol abuse. Moreover, people who smoked $\geq 70$ cigarettes per week for more than 1 year were defined as individuals with a smoking habit.

2.4. SNP Genotyping. All 7 tagSNPs were retrieved from HapMap using the tagger pairwise method in $\mathrm{CHB}$ as follows: $R^{2}$ cutoff $=0.8$ and minor allele frequency (MAF) cutoff $=$ 0.1. Genomic DNA was extracted from whole blood through the standard phenol-chloroform method. Genotyping was performed using the Tm-shift genotyping method [13]. To 
TABLE 1: Baseline characteristics of the investigated participants.

\begin{tabular}{lccc}
\hline Variables & Case & Control & $P$ value \\
\hline Number & 902 & 902 & N/A \\
Male/female & $390 / 512$ & $390 / 512$ & N/A \\
Age $(y)$ & $56.92 \pm 7.36$ & $56.59 \pm 7.43$ & $P=0.45$ \\
TG $(\mathrm{mM})$ & $2.02 \pm 1.68$ & $1.64 \pm 1.12$ & $P<0.01$ \\
HDL $(\mathrm{mM})$ & $1.41 \pm 0.35$ & $1.41 \pm 0.33$ & $P=0.71$ \\
TC $(\mathrm{mM})$ & $5.33 \pm 1.01$ & $5.18 \pm 0.93$ & $P<0.01$ \\
BMI $\left(\mathrm{Kg} / \mathrm{m}^{2}\right)$ & $24.65 \pm 3.25$ & $23.22 \pm 2.88$ & $P<0.01$ \\
Smoking habit & 171 & 147 & $P=0.14$ \\
Alcohol abuse & 152 & 148 & $P=0.80$ \\
\hline
\end{tabular}

TG: triglyceride; HDL: high-density lipoprotein; TC: total cholesterol; BMI: body mass index.

confirm the genotyping results, 100 samples were randomly selected and sequenced with bidirectional coverage by BGI Tech Solutions Company.

2.5. Statistical Analysis. Continuous variables are presented as the mean $\pm \mathrm{SD}$ and analyzed by $t$-test between two groups. Statistical analyses of the allele frequencies between the hypertensive and normotensive subjects and between males and females were performed using the chi-squared test. Logistic regression was used to control the confounding variables. $P$ values, odds ratios (ORs), and 95\% confidence intervals (CIs) were calculated using SPSS 18.0 (SPSS Inc., Chicago, IL, USA). The propensity score analysis was performed using STATA 13.0 according to the method described by Rosenbaum and Rubin [14]. The Hardy-Weinberg equilibrium (HWE) test for genotype distribution was performed for the controls using PEDSTATS [15]. Multifactor dimensionality reduction (MDR), stratified analysis, and crossover analysis were used to identify and characterize interactions among SNPs and nongenetic factors [16]. $P$ values were adjusted for the total number of tested SNPs using the Bonferroni correction method $(\alpha=0.05 / 7 \approx 0.0071)$.

\section{Results}

Table 1 shows the baseline characteristics of the participants. Each group consists of 390 males and 512 females, and the mean ages of the hypertensive participants and controls were similar, demonstrating that the case and control groups were well matched. Serum levels of TC and TG and BMI were significantly higher in the hypertensive groups than those in the control group $(P<0.01)$. However, the serum level of HDL and the percentage of participants with a smoking habit or alcohol abuse were not different between two groups.

Table 2 shows the genotypes of each SNP. The success rate of genotyping was $99 \%$, and all SNPs did not deviate from HWE $(P>0.05)$. Based on the prevalence, OR, and MAF in this study, the genetic power calculator indicated that the sample size is large enough to perform a case-control analysis with $80 \%$ power [17]. According to the $P$ values and ORs, only $\mathrm{G}$ allele of rs17249754 is associated with $\operatorname{EHT}(P=$ 0.005 , OR $(95 \%$ CI $)=1.21(1.06-1.39))$ after correction for multiple testing. However, rs2070759, rs3741895, rs2854371, rs11105357, rs957525, and rs11105358 were not associated with EHT. Inputting all covariates including age, gender, HDL, TC, TG, BMI, smoking habit, and alcohol abuse, the propensity score analysis indicated that still only G allele of rs17249754 is associated with $\mathrm{EHT}(P=0.007, \mathrm{OR}=1.21)$. After control of confounding variables including TC, TG, and BMI, logistic regression also confirmed rs17249754 is associated with EHT $(P=0.007, \mathrm{OR}=1.21)$.

Considering gender difference in EHT [18], the genotyping results were further stratified by gender. Interestingly, both the A allele of rs2070759 and the G allele of rs17249754 were significantly associated with EHT only in women (for rs2070759, $P=0.008$, OR $(95 \% \mathrm{CI})=1.27$ (1.06-1.51); for rs17249754, $P=0.017$, OR (95\% CI) $=1.25$ (1.04-1.49)).

MDR was used to analyze the interaction among SNPs and nongenetic risk factors for EHT, and the software output the best model for "BMI" and "rs2070759, rs17249754, TG, TC, and BMI" with 10/10 cross-validation consistency (Table 3). To determine the manner in which BMI and $A T P 2 B 1$ variants interact to cause hypertension, we performed a stratified analysis. The result showed that when BMI $\geq 25$, neither SNP is associated with hypertension $(P>0.05)$. However, when BMI $<25$, the A allele of rs2070759 or the $\mathrm{G}$ allele of rs17249754 showed a significant association with hypertension (Table 4), indicating that BMI has a major effect and that the ATP2B1 variants have minor effects. Additional crossover analysis also confirmed that BMI had the primary effect (Table 5).

\section{Discussion}

Although dozens of GWASs have been conducted to identify genetic markers for $\mathrm{BP}$ traits or hypertension over the past two decades, ATP2B1 may be the first gene that has been cross-validated in different GWASs. The present study confirmed ATP2B1 variant rs17249754 as strong susceptibility for EHT in the Han Chinese population. The SNP rs17249754 is associated with BP variation and EHT based on several GWASs in different ethnic populations $[1,10,11,19]$, which is also in strong linkage disequilibrium with other genome-wide significant SNPs, such as rs2681472, rs2681492, and rs1105378, within the ATP2B1 gene. Similar findings in different ethnic groups further strengthen the hypothesis that the ATP2B1 gene is a susceptibility locus of likely global significance for BP variation and the development of hypertension.

The ATP2B1 gene encodes the plasma membrane calcium ATPase isoform 1, which plays a critical role in intracellular calcium homeostasis due to its capacity for removing bivalent calcium ions from eukaryotic cells against very large concentration gradients [20]. Although the pathophysiological implications of $A T P 2 B 1$ gene on the development of hypertension are still unclear, results from $A T P 2 B 1$ knockout mouse studies suggested that ATP2B1 may play an important role in the regulation of $\mathrm{BP}$ through alterations of calcium handling and vasoconstriction in vascular smooth muscle cells [21]. ATP2B1 mRNA expression levels in umbilical artery smooth 
TABLE 2: Association statistics for the ATP2B1 variants and hypertension.

\begin{tabular}{|c|c|c|c|c|c|c|c|c|c|}
\hline SNP & Genotype & Group & & enoty & & MAF & $P$ value & OR & $95 \% \mathrm{CI}$ \\
\hline \multirow{6}{*}{ rs3741895 } & \multirow{6}{*}{$\mathrm{AA} / \mathrm{AG} / \mathrm{GG}$} & Case & 778 & 122 & 0 & 0.07 & \multirow{2}{*}{0.954} & \multirow{2}{*}{0.99} & \multirow{2}{*}{$0.77-1.29$} \\
\hline & & Control & 778 & 121 & 0 & 0.07 & & & \\
\hline & & Male case & 331 & 58 & 0 & 0.07 & \multirow{2}{*}{0.433} & \multirow{2}{*}{0.86} & \multirow{2}{*}{$0.58-1.27$} \\
\hline & & Male control & 338 & 50 & 0 & 0.06 & & & \\
\hline & & Female case & 447 & 64 & 0 & 0.06 & \multirow{2}{*}{0.533} & \multirow{2}{*}{1.12} & \multirow{2}{*}{$0.79-1.59$} \\
\hline & & Female control & 440 & 71 & 0 & 0.07 & & & \\
\hline \multirow{6}{*}{ rs2854371 } & \multirow{6}{*}{ CC/CT/TT } & Case & 519 & 339 & 44 & 0.24 & \multirow{2}{*}{0.879} & \multirow{2}{*}{1.01} & \multirow{2}{*}{$0.87-1.18$} \\
\hline & & Control & 510 & 347 & 41 & 0.24 & & & \\
\hline & & Male case & 226 & 143 & 21 & 0.24 & \multirow{2}{*}{0.648} & \multirow{2}{*}{0.95} & \multirow{2}{*}{$0.75-1.20$} \\
\hline & & Male control & 226 & 146 & 15 & 0.23 & & & \\
\hline & & Female case & 293 & 196 & 23 & 0.24 & \multirow{2}{*}{0.553} & \multirow{2}{*}{1.06} & \multirow{2}{*}{$0.87-1.30$} \\
\hline & & Female control & 284 & 201 & 26 & 0.25 & & & \\
\hline \multirow{6}{*}{ rs2070759 } & & Case & 266 & 453 & 183 & 0.45 & $0036^{*}$ & 115 & $101-131$ \\
\hline & & Control & 223 & 476 & 203 & 0.49 & 0.050 & 1.10 & $1.01-1.31$ \\
\hline & А А /АC/CC & Male case & 107 & 201 & 82 & 0.47 & 0879 & 102 & $083-124$ \\
\hline & АR/ & Male control & 109 & 194 & 87 & 0.47 & 0.077 & 1.02 & $0.03-1.24$ \\
\hline & & Female case & 159 & 252 & 101 & 0.44 & $0008 *$ & 127 & $106-151$ \\
\hline & & Female control & 114 & 282 & 116 & 0.50 & 0.000 & 1.27 & $1.00-1.01$ \\
\hline & & Case & 722 & 172 & 8 & 0.10 & 0472 & 092 & $074-115$ \\
\hline & & Control & 733 & 163 & 6 & 0.10 & & & \\
\hline rs11105357 & $\mathrm{CC} / \mathrm{CT} / \mathrm{TT}$ & Male case & 320 & 64 & 6 & 0.10 & 0.407 & 1.15 & $0.83-1.59$ \\
\hline & & Male control & 309 & 76 & 5 & 0.11 & & & \\
\hline & & Female case & 402 & 108 & 2 & 0.11 & 0.088 & 0.78 & $0.58-1.04$ \\
\hline & & Female control & 424 & 87 & 1 & 0.09 & 0.088 & 0.18 & $0.58-1.04$ \\
\hline & & Case & 546 & 314 & 40 & 0.22 & 0.705 & 1.03 & $0.88-1.21$ \\
\hline & & Control & 541 & 313 & 45 & 0.22 & & & \\
\hline rs957525 & $\mathrm{AA} / \mathrm{AG} / \mathrm{GG}$ & Male case & 245 & 132 & 12 & 0.20 & 0239 & 116 & 0 91-148 \\
\hline & & Male control & 231 & 141 & 17 & 0.22 & & & \\
\hline & & Female case & 301 & 182 & 28 & 0.23 & 0615 & 095 & $077-117$ \\
\hline & & Female control & 310 & 172 & 28 & 0.22 & 0.615 & 0.95 & $0 . / /-1.1 /$ \\
\hline & & Case & 43 & 311 & 547 & 0.22 & 0160 & 112 & $096-132$ \\
\hline & & Control & 35 & 293 & 574 & 0.20 & & & \\
\hline rc11105358 & CC/CG/GG & Male case & 24 & 124 & 242 & 0.22 & 0903 & 102 & $080-129$ \\
\hline & & Male control & 18 & 134 & 238 & 0.22 & & & \\
\hline & & Female case & 19 & 187 & 305 & 0.22 & 0.076 & 122 & $098-151$ \\
\hline & & Female control & 17 & 159 & 336 & 0.19 & & & \\
\hline & & Case & 102 & 417 & 383 & 0.34 & $0.005^{*}$ & 0.82 & $0.72-0.94$ \\
\hline & & Control & 143 & 416 & 343 & 0.39 & 0.005 & 0.82 & $0.12-0.94$ \\
\hline $\operatorname{rs} 17249754$ & $\mathrm{AA} / \mathrm{AG} / \mathrm{G} G$ & Male case & 46 & 182 & 162 & 0.35 & 0128 & 085 & $069-1.05$ \\
\hline $181 / 249734$ & AA/AU/UU & Male control & 59 & 185 & 146 & 0.39 & & & \\
\hline & & Female case & 56 & 235 & 221 & 0.34 & $0017^{*}$ & 080 & $067-096$ \\
\hline & & Female control & 84 & 231 & 197 & 0.39 & & & \\
\hline
\end{tabular}

$P$ values were obtained from the comparison of two allele frequencies. OR: odds ratio; CI: confidence interval. ${ }^{*} P$ value was less than 0.05 .

TABLE 3: MDR analysis of gene-environment interaction.

\begin{tabular}{lccc}
\hline Best model & Testing odds ratio & Testing $X^{2}$ & Cross-validation consistency \\
\hline BMI & $2.25(95 \%$ CI: $1.19-4.24)$ & $6.36(P=0.012)$ & $10 / 10$ \\
BMI, TG & $2.00(95 \%$ CI: $1.10-3.61)$ & $5.27(P=0.021)$ & $9 / 10$ \\
rs2070759, rs17249754, TG, TC, and BMI & $1.83(95 \%$ CI: $1.01-3.30)$ & $4.05(P=0.044)$ & $10 / 10$ \\
\hline
\end{tabular}


TABLE 4: Stratified analysis of interaction between BMI and ATP2B1 variants.

\begin{tabular}{|c|c|c|c|c|c|c|c|c|c|}
\hline SNP & Genotype & BMI & Group & & Numbe & & $P$ value & OR & $95 \% \mathrm{CI}$ \\
\hline \multirow{4}{*}{ rs2070759 } & \multirow{4}{*}{$\mathrm{AA} / \mathrm{AC} / \mathrm{CC}$} & \multirow{2}{*}{$<25$} & Case & 147 & 268 & 104 & \multirow{2}{*}{$0.022^{*}$} & \multirow{2}{*}{1.21} & \multirow{2}{*}{$1.03-1.42$} \\
\hline & & & Control & 156 & 359 & 164 & & & \\
\hline & & \multirow{2}{*}{$\geq 25$} & Case & 115 & 185 & 83 & \multirow{2}{*}{0.349} & \multirow{2}{*}{0.89} & \multirow{2}{*}{$0.71-1.13$} \\
\hline & & & Control & 70 & 114 & 39 & & & \\
\hline \multirow{4}{*}{ rs17249754 } & \multirow{4}{*}{ AA/AG/GG } & \multirow{2}{*}{$<25$} & Case & 59 & 241 & 219 & \multirow{2}{*}{$0.011^{*}$} & \multirow{2}{*}{0.80} & \multirow{2}{*}{$0.68-0.95$} \\
\hline & & & Control & 104 & 331 & 244 & & & \\
\hline & & \multirow{2}{*}{$\geq 25$} & Case & 43 & 176 & 164 & \multirow{2}{*}{0.61} & \multirow{2}{*}{0.94} & \multirow{2}{*}{$0.74-1.20$} \\
\hline & & & Control & 37 & 85 & 101 & & & \\
\hline
\end{tabular}

$P$ values were obtained from the comparison of two allele frequencies. OR: odds ratio; CI: confidence interval.

${ }^{*} P$ value was less than 0.05 .

TABLE 5: Crossover analysis of interaction between BMI and ATP2B1 variants.

\begin{tabular}{lccccccc}
\hline SNP & BMI & Allele & Case & Control & $P$ value & OR & $95 \%$ CI \\
\hline \multirow{2}{*}{ rs2070759 } & $<25$ & C & 476 & 687 & 1 & 1 & NA \\
& $<25$ & A & 562 & 671 & $0.022^{*}$ & 1.21 & $1.03-1.42$ \\
& $\geq 25$ & C & 351 & 192 & $P<0.001^{*}$ & 0.38 & $0.31-0.47$ \\
& $\geq 25$ & A & 415 & 254 & $P<0.001^{*}$ & 0.42 & $0.35-0.52$ \\
\hline & $<25$ & A & 359 & 539 & 1 & 1 & NA \\
rs17249754 & $<25$ & G & 679 & 819 & $0.011^{*}$ & 0.80 & $0.68-0.95$ \\
& $\geq 25$ & A & 262 & 159 & $P<0.001^{*}$ & 0.40 & $0.32-0.51$ \\
& $\geq 25$ & G & 504 & 287 & $P<0.001^{*}$ & 0.38 & $0.31-0.46$ \\
\hline
\end{tabular}

$P$ values were obtained from the comparison of two allele frequencies. OR: odds ratio; CI: confidence interval.

${ }^{*} P$ value was less than 0.05 .

muscle cells were found to be significantly different among rs11105378 genotypes, which may be a potential mechanism by which changes in the ATP $2 B 1$ gene product levels are involved in BP regulation [1]. According to HapMap CHB, rs17249754 and rs1105378 are in strong linkage disequilibrium $\left(D^{\prime}=1, r^{2}=0.95\right)$ in Chinese populations; therefore, rs17249754 was genotyped instead of rs1105378 in the present study. In our replication study, we also found that rs1105378 is significantly associated with hypertension $(P<0.01)$. Therefore, the SNPs rs2681472, rs2681492, and rs17249754 are in strong linkage disequilibrium with rs1105378 and may be a genetic marker for the development of hypertension, whereas rs1105378 may have a biological function.

Another finding of the present study is that ATP2B1 variants are associated with EHT only in women. According to the World Health Organization's (WHO) "Global Status Report on Noncommunicable Diseases 2014" (http://www .who.int/nmh/publications/ncd-status-report-2014/en/), hypertension occurs at a lower rate and at a later age in females than males in all WHO regions. The impact of gender on the prevalence, presentation, and long-term outcome of hypertension has long been a topic of active research. Recent data from several large epidemiological studies showed that awareness, treatment, and control rates of hypertension are higher among women than men, which may cause the gender difference in hypertension [22, 23]. The pathophysiological mechanisms underlying the disparity in blood pressure levels between the two genders are poorly defined, although many hypotheses have been proposed, with hormonal hypotheses prevailing [24]. Similar to our study, several previous studies also found a gender-specific association between gene polymorphisms and EHT [25-27]. Therefore, further basic research is of paramount importance to uncover the genetic and biological mechanisms mediating potential gender differences in hypertension.

EHT is a typical complex disease [28], with dozens of risk factors, such as obesity, physical inactivity, high-fat diet, cigarette smoking, alcohol abuse, excessive salt intake, and mental stress [29-31]. Growing evidence indicates that interactions among multiple genes and environmental factors may increase the susceptibility to EHT [32]. Our previous study has shown that interaction analysis may provide somewhat more information than a single genetic association study $[12,33]$. In the present study, MDR analysis demonstrated that $\mathrm{BMI}$ itself and the interaction between $A T P 2 B 1$ variants and $\mathrm{BMI}$ increase the susceptibility to hypertension. Because BMI represents the internal metabolic status and physiological environment [34], it is not surprising that BMI has a major effect in the development of hypertension, while the ATP2B1 variants have a minor effect. With the development of statistical methods for the evaluation of gene-gene and geneenvironment interactions, more missing inheritability will be identified and more specific mechanisms will be discovered $[35,36]$.

In conclusion, we confirmed the association of ATP2B1 variants with the susceptibility to EHT in the Han Chinese population, especially in the females. Moreover, the interaction of BMI and ATP2B1 variants increased the susceptibility to hypertension, with BMI having a major effect and ATP2B1 variants having a minor effect.

\section{Conflict of Interests}

The authors declare no conflict of interests.

\section{Authors' Contribution}

Jin Xu, Hai-xia Qian, and Su-pei Hu contributed equally to this work. 


\section{Acknowledgments}

This research was supported by the grants from the National Natural Science Foundation of China (81402747), Zhejiang Natural Science Foundation (LQ13C060001), Analysis and Measurement Foundation of Zhejiang Province (2014C37047), Scientific Research Fund of Zhejiang Provincial Education Department (Y201224146), and the K.C. Wong Magna Fund in Ningbo University.

\section{References}

[1] Y. Tabara, K. Kohara, Y. Kita et al., "Common variants in the ATP2B1 gene are associated with susceptibility to hypertension: the Japanese millennium genome project," Hypertension, vol. 56, no. 5, pp. 973-980, 2010.

[2] O. A. Carretero and S. Oparil, "Essential hypertension. Part I: definition and etiology," Circulation, vol. 101, no. 3, pp. 329-335, 2000.

[3] G. B. Ehret, "Genome-wide association studies: contribution of genomics to understanding blood pressure and essential hypertension," Current Hypertension Reports, vol. 12, no. 1, pp. 17-25, 2010.

[4] S. Rafiq, S. Anand, and R. Roberts, "Genome-wide association studies of hypertension: have they been fruitful?" Journal of Cardiovascular Translational Research, vol. 3, no. 3, pp. 189-196, 2010.

[5] G. B. Ehret, P. B. Munroe, K. M. Rice et al., "Genetic variants in novel pathways influence blood pressure and cardiovascular disease risk," Nature, vol. 478, no. 7367, pp. 103-109, 2011.

[6] P. G. Joseph, G. Pare, and S. S. Anand, "Exploring geneenvironment relationships in cardiovascular disease," Canadian Journal of Cardiology, vol. 29, no. 1, pp. 37-45, 2013.

[7] D. Levy, G. B. Ehret, K. Rice et al., "Genome-wide association study of blood pressure and hypertension," Nature Genetics, vol. 41, no. 6, pp. 677-687, 2009.

[8] C. Newton-Cheh, T. Johnson, V. Gateva et al., "Genome-wide association study identifies eight loci associated with blood pressure," Nature Genetics, vol. 41, no. 6, pp. 666-676, 2009.

[9] N. Hirawa, A. Fujiwara, and S. Umemura, "ATP2B1 and blood pressure: from associations to pathophysiology," Current Opinion in Nephrology \& Hypertension, vol. 22, no. 2, pp. 177-184, 2013.

[10] Y. S. Cho, M. J. Go, Y. J. Kim et al., "A large-scale genome-wide association study of Asian populations uncovers genetic factors influencing eight quantitative traits," Nature Genetics, vol. 41, no. 5, pp. 527-534, 2009.

[11] N. Kato, F. Takeuchi, Y. Tabara et al., "Meta-analysis of genomewide association studies identifies common variants associated with blood pressure variation in east Asians," Nature Genetics, vol. 43, no. 6, pp. 531-538, 2011.

[12] L. Ji, X. Cai, L. Zhang et al., "Association between polymorphisms in the renin-angiotensin-aldosterone system genes and essential hypertension in the Han Chinese population," PLoS ONE, vol. 8, no. 8, Article ID e72701, 2013.

[13] J. Wang, K. Chuang, M. Ahluwalia et al., "High-throughput SNP genotyping by single-tube PCR with Tm-shift primers," BioTechniques, vol. 39, no. 6, pp. 885-893, 2005.

[14] P. R. Rosenbaum and D. B. Rubin, "The central role of the propensity score in observational studies for causal effects," Biometrika, vol. 70, no. 1, pp. 41-55, 1983.
[15] J. E. Wigginton and G. R. Abecasis, "PEDSTATS: descriptive statistics, graphics and quality assessment for gene mapping data," Bioinformatics, vol. 21, no. 16, pp. 3445-3447, 2005.

[16] A. A. Motsinger and M. D. Ritchie, "The effect of reduction in cross-validation intervals on the performance of multifactor dimensionality reduction," Genetic Epidemiology, vol. 30, no. 6, pp. 546-555, 2006.

[17] S. Purcell, S. S. Cherny, and P. C. Sham, "Genetic power calculator: design of linkage and association genetic mapping studies of complex traits," Bioinformatics, vol. 19, no. 1, pp. 149150, 2003.

[18] M. Doumas, V. Papademetriou, C. Faselis, and P. Kokkinos, "Gender differences in hypertension: myths and reality," Current Hypertension Reports, vol. 15, no. 4, pp. 321-330, 2013.

[19] T. N. Kelly, F. Takeuchi, Y. Tabara et al., "Genome-wide association study meta-analysis reveals transethnic replication of mean arterial and pulse pressure loci," Hypertension, vol. 62, no. 5, pp. 853-859, 2013.

[20] S. Olson, M. G. Wang, E. Carafoli, E. E. Strehler, and O. W. McBride, "Localization of two genes encoding plasma membrane $\mathrm{Ca}^{2+}$-transporting ATPases to human chromosomes 1q25-32 and 12q21-23," Genomics, vol. 9, no. 4, pp. 629-641, 1991.

[21] Y. Kobayashi, N. Hirawa, Y. Tabara et al., "Mice lacking hypertension candidate gene ATP2B1 in vascular smooth muscle cells show significant blood pressure elevation," Hypertension, vol. 59, no. 4, pp. 854-860, 2012.

[22] A. S. Go, D. Mozaffarian, V. L. Roger et al., "Heart disease and stroke statistics-2013 update: a report from the American Heart Association," Circulation, vol. 127, no. 1, pp. e6-e245, 2013.

[23] B. M. Egan, Y. Zhao, and R. N. Axon, "US trends in prevalence, awareness, treatment, and control of hypertension, 1988-2008," The Journal of the American Medical Association, vol. 303, no. 20, pp. 2043-2050, 2010.

[24] K. Tsuda, "Roles of sex steroid hormones and nitric oxide in the regulation of sympathetic nerve activity in women," Hypertension, vol. 61, no. 4, article e36, 2013.

[25] K. Dhanachandra Singh, A. Jajodia, H. Kaur, R. Kukreti, and M. Karthikeyan, "Gender specific association of RAS gene polymorphism with essential hypertension: a case-control study," BioMed Research International, vol. 2014, Article ID 538053, 10 pages, 2014.

[26] W. Zhao, Y. Wang, L. Wang et al., "Gender-specific association between the kininogen 1 gene variants and essential hypertension in Chinese Han population," Journal of Hypertension, vol. 27, no. 3, pp. 484-490, 2009.

[27] R. Periaswamy, U. Gurusamy, D. G. Shewade et al., "Gender specific association of endothelial nitric oxide synthase gene (Glu298Asp) polymorphism with essential hypertension in a south Indian population," Clinica Chimica Acta, vol. 395, no. 1-2, pp. 134-136, 2008.

[28] G. W. Pickering, H. Keen, G. Rose, and A. Smith, "The nature of essential hypertension," The Lancet, vol. 274, no. 7110, pp. 10271030, 1959.

[29] T. A. Kotchen, "Obesity-related hypertension: epidemiology, pathophysiology, and clinical management," American Journal of Hypertension, vol. 23, no. 11, pp. 1170-1178, 2010.

[30] T. J. Wang and R. S. Vasan, "Epidemiology of uncontrolled hypertension in the United States," Circulation, vol. 112, no. 11, pp. 1651-1662, 2005.

[31] M. J. Horan and C. Lenfant, "Epidemiology of blood pressure and predictors of hypertension," Hypertension, vol. 15, supplement 2, pp. I20-I24, 1990. 
[32] J. Kuneš and J. Zicha, "Developmental windows and environment as important factors in the expression of genetic information: a cardiovascular physiologist's view," Clinical Science, vol. 111, no. 5, pp. 295-305, 2006.

[33] J. Xu, L.-D. Ji, L.-N. Zhang et al., "Lack of association between STK39 and hypertension in the Chinese population," Journal of Human Hypertension, vol. 27, no. 5, pp. 294-297, 2013.

[34] R.-N. Feng, C. Zhao, C. Wang et al., "BMI is strongly associated with hypertension, and waist circumference is strongly associated with type 2 diabetes and dyslipidemia, in Northern Chinese adults," Journal of Epidemiology, vol. 22, no. 4, pp. 317-323, 2012.

[35] B. Mukherjee, J. Ahn, S. B. Gruber, and N. Chatterjee, “Testing gene-environment interaction in large-scale case-control association studies: possible choices and comparisons," American Journal of Epidemiology, vol. 175, no. 3, pp. 177-190, 2012.

[36] R. Kazma, M.-C. Babron, and E. Génin, "Genetic association and gene-environment interaction: a new method for overcoming the lack of exposure information in controls," American Journal of Epidemiology, vol. 173, no. 2, pp. 225-235, 2011. 


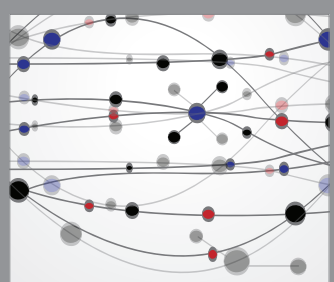

The Scientific World Journal
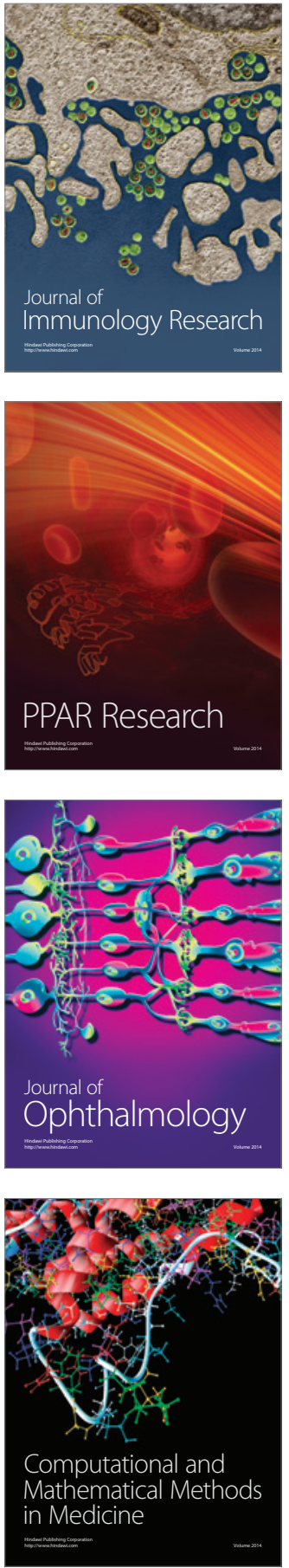

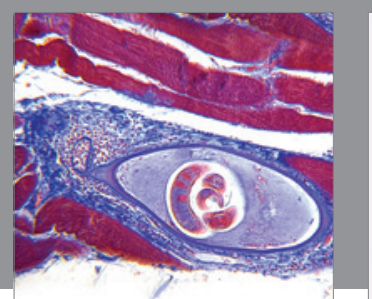

Gastroenterology Research and Practice

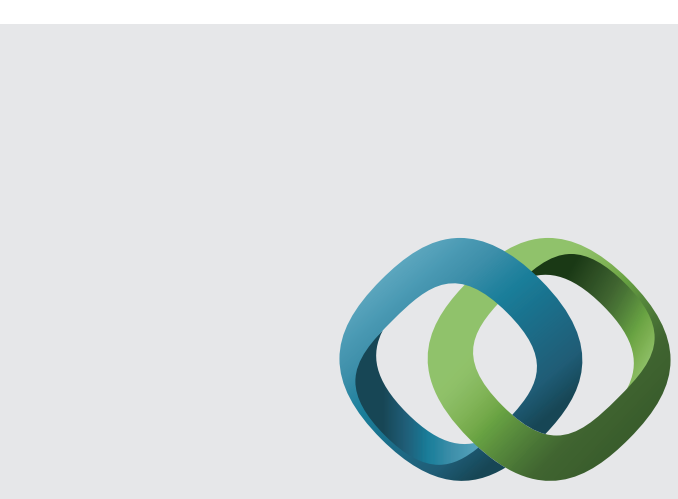

\section{Hindawi}

Submit your manuscripts at

http://www.hindawi.com
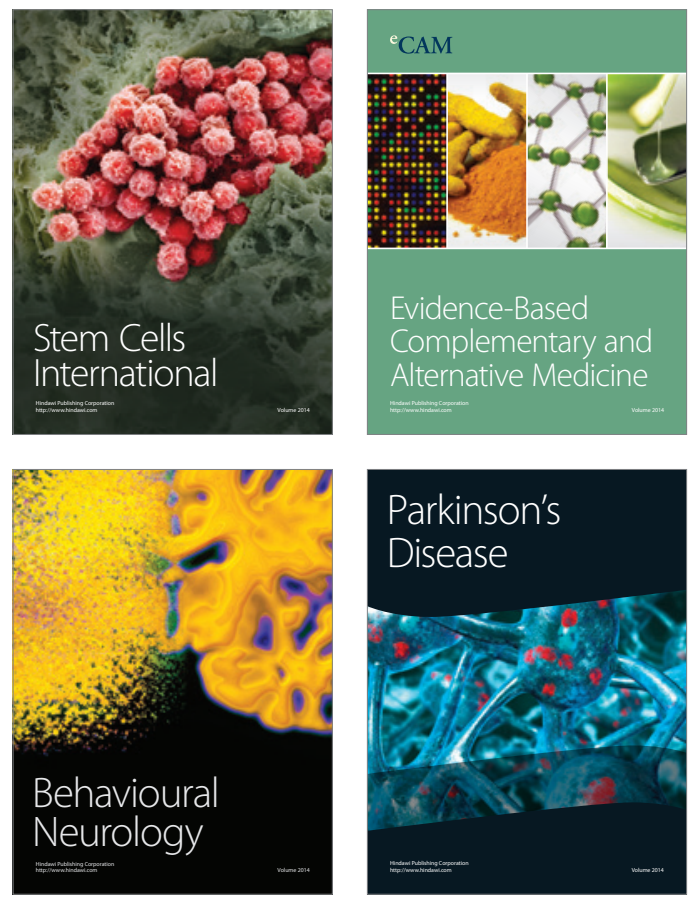
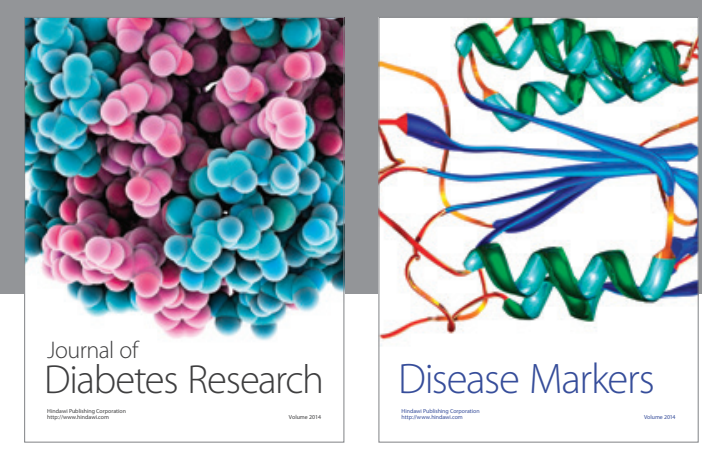

Disease Markers
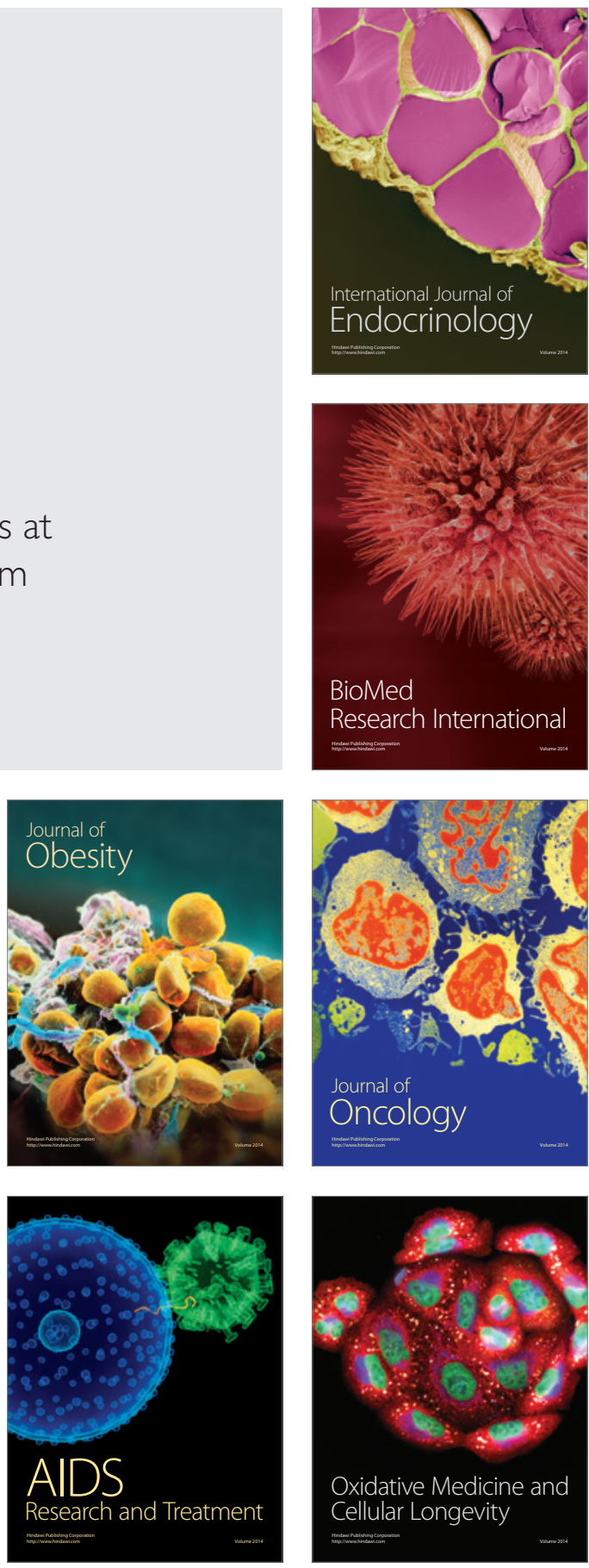